\title{
Detection of Diarrheagenic Pathogens in Improved Water Using PCR-Based Specific Techniques in Low- and Middle-Income Countries: A Systematic Review and Meta-Analysis Protocol
}

Shibabaw Tadesse Gemeda ( $\square$ shibabawt@gmail.com )

Addis Ababa University https://orcid.org/1234-5678-9123-4567

Negasa Eshete Soboksa

Ethiopian Institute of Water Resources, Addis Ababa University, Ethiopia

Adey Feleke Desta

Division of Environmental Biotechnolgoy, Institute of Biotechnology, Addis Ababa Univerisity

Sirak Robele Gari

Ethiopian Institute of Water Resources, Addis Ababa University

\section{Protocol}

Keywords: Diarrheagenic pathogens, improved water, Polymerase chain reaction, Low- and middleincome countries

Posted Date: August 10th, 2020

DOI: https://doi.org/10.21203/rs.3.rs-55533/v1

License: (1) This work is licensed under a Creative Commons Attribution 4.0 International License.

Read Full License 


\section{Abstract}

Background: Effective application of Polymerase Chain Reaction (PCR) based methods in detecting pathogenic microorganisms in improved water samples were limited in low- and middle-income countries. This limitation initiated this systematic review to assess the effective characterization method that could be applied in low- and middle-income countries. Therefore, the aim of this review and analysis is to pullout the available evidences in characterization of pathogenic microorganisms in improved drinking water that could be effectively applied in low- and middle-income countries.

Methods: We will search for published literatures using Google Scholar, PubMed or Medline, Scopus and Cochrane Library databases to explore the various PCR based methods recommended for the detection and enumeration of pathogens to examine quality of drinking water provided for communities. All studies conducted in low- and middle-income countries that use any types of PCR methods in water samples without publication date restriction were included in the review. The primary outcome of this study is assessing the magnitude of diarrheagenic pathogens detected in improved water supplies by PCR application in low- and middle-income countries. Critical appraisal will be conducted on all papers selected for inclusion in the review. The studies selected will be assessed by the reviewers for retrieval prior to inclusion in the review.

Discussion: This review will systematically discover and integrate the evidence available on the detection of diarrheagenic pathogens through the application of PCR based methods for water quality determination. This facilitates water quality monitoring activities. In this review, information about the applied PCR method, detected diarrheagenic pathogens, water sample size, effectiveness in terms of time and cost, will be gathered and summarized. The findings from this review and analysis will provide compiled data on the magnitude of the detected diarrheagenic pathogenic microbes by type and amount in improved water supply through the application of PCR techniques in low- and middle-income countries. It is envisaged that the study will promote the application of PCR methods for drinking water quality monitoring in these low- and middle-income countries.

\section{Background}

Genetic diversity of human enteric bacterial [1],viral [2-4]and protozoal genomes in water samples are becoming increasing and complicated as a result of contamination of improved water at the sources, collection and storage at home that creates increasing public health threats in low- and middle-income countries. The detection of human enteric viruses in drinking water confirms the quality limits for indicator bacteria that warrants an investigation of the potential transmission risk of infection constituted by different waterborne genomes of viruses [5, 6]and protozoans [7].

To secure water safety, identifying effective methods of characterizing variety of pathogens in water samples beyond bacterial presence/absence and colony count is important [8]. Studies show that false positive results by different techniques of pathogenic microbes' detection methods [9-13] in water 
samples mislead the actions to be taken to improve the water quality. Thus, fresh perspectives on molecular technique based on polymerase chain reaction (PCR) was applied on drinking water since 2003 followed by identifying the microbiome in human gut in 2006 [14]. The methods are ranged from detecting up to quantification of bacterial, viral and protozoal pathogens in water samples by using small volumes of samples usually 1 or 2 microliter $(\mu \mathrm{L})$ [15].

Even though, the molecular method is still under refinement to standardize [16], the most frequently used nucleic-acid-based methods for coliform detection in drinking water are the PCR methods $[14,17]$. Those methods are in general an emerging approach [14] with different methods from, quantitative-PCR (Q$P C R$ ), real-time reverse transcription polymerase chain reaction (RT-qPCR) [18], conventional PCR, real-time PCR (RT-PCR), multiplex PCR (mPCR), competitive PCR (cPCR), Serial multistandard-assisted (SmaRTPCR) up to $16 S$ rRNA gene sequencing[14, 19-21] that can be applied in water samples. PCR methods are powerful molecular biology techniques for the detection of target DNA in various samples and for the detection of many kinds of pathogens. PCR ,compared to culture methods, increases the overall detection frequency of the bacterial enteropathogens by $4 \%$ can measure specific gene expressions and can detect enteric viruses and viable but nonculturable (VBNC) pathogens in water samples [18, 22].

However, this development in method of identification and quantification of pathogens in water samples were not up taken by the low- and middle-income countries as required despite molecular assays are needed for broad access in the developing world [23] for comprehensive and accurate assessments of water quality [24]. There are advances in pathogenic microbial testing methods. As a result new understandings about newly emerged waterborne diarrheal pathogens are happening. Thus, reports show increase of different genotypes of microorganisms in number [25]. These multiple factors pushes towards the interest of this work that primarily focus on the assessment of the magnitude of diarrheagenic pathogenic microbes in water by application of molecular method/s in low- and middleincome countries.

Further, there is no a previous review that provide organized data about the magnitude of diarrheagenic pathogens in improved water samples using PCR detection and enumeration techniques in low- and middle-income countries. As a result, this systematic review will assess the magnitude of detected and enumerated diarrheagenic pathogens in improved water by PCR techniques in low- and middle-income countries. This contribute for the improvement of the water quality monitoring that leads to the improvement of public health in regard to water-borne communicable diseases in low- and middle-income countries through the application of PCR techniques in regular water quality monitoring.

\section{Objective}

The objective of this systematic review and meta-analysis will be to pool out the available evidence on the detection of diarrheagenic pathogens in improved water using PCR-based specific techniques in lowand middle-income countries. The evidences will be in terms of detected pathogens; it's magnitude, gene types, amount, number of copies and health impact. 


\section{Research Question}

The research question of this review is "What is/are the magnitude and gene types of the major diarrheagenic pathogens detected in improved water samples by the application of PCR method/s that are potential health risks for the people in low- and middle-income countries?"

\section{Methods And Materials}

\section{Study design and protocol}

This systematic review protocol follows the Preferred Reporting Items for Systematic Review and MetaAnalysis Protocols (PRISMA-P) statements [26].

\section{Criteria for considering studies for this review}

Studies that meet the following criteria will be included in this review.

\section{Types of studies}

All studies done to characterize diarrheagenic pathogens using PCR techniques applied on improved water samples in low- and middle-income countries will be included in this review and analysis.

\section{Participants}

The review and analysis will include all studies carried out on improved water samples to characterize any type of diarrheagenic microbes using the available PCR techniques in low- and middle-income countries without publication year restriction.

\section{Interventions and comparator}

This review and analysis will consider studies conducted to characterize diarrheagenic pathogens in improved water samples using PCR techniques as characterization method of pathogenic microbes. Studies that include the detected pathogens; gene numbers/mean/average/maximum and minimum, copies, types, detection capacity and health impact will be considered. The operational definitions of the molecular approach: polymerase chain reaction methods (PCR): ISO 13136:2012 is a technique in molecular genetics that permits the analysis of any short sequence of DNA (or RNA) in samples containing only minute quantities of DNA or RNA. PCR is used to reproduce (amplify) selected sections of DNA or RNA for analysis [27].

\section{Types of outcome measures}

This review and analysis will consider studies that include diarrhea causing microbe's type, numbers, gene copies, health impacts and detection capacity. The primary outcome of this study will be assessing of the magnitude of diarrhea causing microbes in improved water using PCR characterization method in low- and middle-income countries. 


\section{Information sources and search strategy}

The literature search consists of electronic database and hand searches that will be carried out to identify appropriate peer-reviewed articles that meet the inclusion criteria. We will search for published literatures using PubMed/Medline, Scopus and Cochrane Library electronic databases and Google Scholar hand searches for important journals, to explore the various diarrheagenic microbes detected using PCR-based methods to examine quality of drinking water provided for communities in low- and middle-income countries. References to other literatures will also be considered, making use of snowball techniques. The search strategy will be conducted using major and broad terms limited to studies published literatures in English language. All studies conducted in low- and middle-income countries that use any types of PCR method on improved water samples without publication date restriction will be included in the review and analysis. We will use the initial search terms of the following keywords in combination with medical subject headings (MeSH) terms: (Polymerase chain reaction or PCR) AND (Characterization OR Identification) AND (Improved water OR Drinking water) AND (Pathogenic microbes OR E. coli OR Bacteria OR Virus OR Protozoa) AND (Low- and middle-income countries OR Africa OR Asia OR Caribbean Countries). A full search strategy for PubMed/Medline and Cochrane databases is detailed in Table 1. The results of the search and the full process for selecting included studies will be reported in full in the final report and presented in a PRISMA flow diagram [26] (Fig. 1).

Table 1

Systematic review search terms and strategy for Pubmed/Medline databases of main terms.

\section{S. Query \\ No}

1. "polymerase chain reaction"[MeSH] OR Polymerase chain reaction[tw] OR PCR(tw)

2. Detection (tw) OR Identification (tw) OR Characterization(tw)

3. Pathogenic microbes (tw) OR "bacteria"[MeSH Terms] OR Bacteria[Text Word] OR "escherichia coli"[MeSH Terms] OR E. coli[Text Word]OR "viruses"[MeSH Terms] OR Virus[Text Word]OR Protozoa (tw)

4. Improved water (tw) OR "drinking water"[MeSH] OR Drinking water[tw]

5. Low- and middle-income countries (tw) OR Africa (tw) OR Asia (tw) OR Caribbean Countries (tw)

6. 1 AND 2 AND 3 AND 4 AND 5

7. Limit 6to English

$\mathrm{MeSH}=$ Medical subject headings, $\mathrm{tw}=$ text word

\section{Data collection and analysis}

Data management and selection of studies 
Data collection will be carried out using developed excel spreadsheet tool. The searched results will be managed using the Mendeley Desktop reference management software version 1.19.4 (Mendeley Ltd., Elsevier, Netherlands). The screening of studies will be conducted by two independent reviewers (STG and NES). The articles found by searches in databases and hand searches will be evaluated for inclusion at three levels. That is, by title, then by abstract, and finally by the full text. The full text of selected studies will be retrieved and assessed in detail against the inclusion criteria. Discrepancies will be discussed between reviewers and refine inclusion criteria. For the screening of articles at full text level, rejection of an article will be decided by the review team upon suggestion of the first reader.

Details regarding the final decision of inclusion of articles will be clarified and archived in a database. In cases of uncertainty in the decision to include or exclude an article, the reviewer will include this article for the next level of screening. The documents without abstracts will be screened at the full text level. A list of articles excluded at full text level will be provided in the systematic review and accompanied by reasons for exclusion.

\section{Data extraction and management}

Data will be extracted and collected by two independent reviewers (STG and NES) from studies included in the review using a prepared data extraction excel spreadsheet tool. For each study, authors' name, place and year of publication, study period, date of search, and the study conducted country, sample type, data on sample size, types of PCR techniques used, result of included studies: detected type of pathogen/s, amount, detection capacity and health impact will be extracted. The data extraction form will be pretested and revised.

Risk of bias of included studies

Two authors (STG and NES) will evaluate independently the methodological quality of the studies that meet the selection criteria. The methodological quality of all studies that meet the selection criteria will be evaluated independently by two authors using the Joanna Briggs Institute (JBI) Critical Appraisal tools [28]. Each study will be assessed individually and independently by the two reviewers, both at the outcome and study level to generate an overall risk of bias score. The reviewers will judge each study as critical, serious, moderate, or low risk of bias via the assessment of the gathered information. The rating for each bias criterion of the two authors will then be compared. Disagreements between the two authors on individual bias criterion will be identified and discussed in an attempt to reach a consensus. Any disagreements that arise between the reviewers will be resolved through discussion or with a third and/or fourth reviewer/s (SRG and AFD).

Dealing with missing data

The reviewers will contact the authors for missing data and clarification of primary studies if required; such inclusions will be reported in the review. We will reject if the author/s didn't respond about the missing data and clarification request. 
The reviewers will conduct a narrative synthesis first to describe study details, participant and intervention characteristics, and outcomes of the included studies. STATA version-16(College Station, TX 77845 , USA) will be used for the data synthesis. We will calculate $95 \%$ confidence intervals and $p$-values for the outcome.

Investigation of sources of heterogeneity

Heterogeneity will be assessed statistically using chi-square test or Q-test statistics for presence of heterogeneity and inverse variance index $\left(1^{2}\right)$ for magnitude of heterogeneity. Variance index values will be classified as follows: no relevant heterogeneity ( $0-25 \%)$, moderate heterogeneity $(26-50 \%)$, and significant heterogeneity $(>50 \%)$ [29]. Forest plots will be generated to present the pooled estimates to summarize where there are two or more similar studies with similar outputs. Funnel plots of the result of studies will be evaluated for publication bias. Sensitivity analyses will be repeated after exclusion of studies with a high risk of bias. The presence of publication bias will be examined using a funnel plot, Egger's and Begg's test. A sensitivity analysis will be repeated after excluding one study to observe the impact of the individual study on the pooled estimate.

Assessment of the quality of the evidence

The quality of evidence of the outcomes will be assessed with the Grading of Recommendations, Assessment, Development, and Evaluation (GRADE) approach using GRADEPro GDT version 3.6.1 / 2019 (McMaster University, ON, Canada [30, 31]. According to GRADE, evidence quality assessment is performed for each outcome, and the combined available evidence is considered. The GRADE approach will classify the quality of the evidence into four levels: high, moderate, low, and very low based on the comprehensive assessment of inconsistency, indirect evidence (not generalizable), inaccuracy, and publication bias. These levels represent confidence in the estimation of the treatment effects presented. The level of evidence and strength of recommendation will be determined by discussion involving all authors.

\section{Discussion}

This review will systematically explore and fit in the evidence available on the detection of diarrheagenic pathogens in improved water samples by the application of the PCR methods in low- and middle-income countries. In this review, information about the types of PCR techniques used, result of included studies: detected type of pathogen/s, amount, detection capacity and health impact will be gathered and summarized. The review will be reported according to the PRISMA guidelines [26] and submitted to appropriate journal for publication. The findings from this study will provide directions for future research and improve water quality monitoring with an understanding of the importance of PCR application in informing the magnitude of pathogens in low- and middle-income countries. Only peer reviewed English language publications will be included and this would be the limitation of this review. 


\section{Abbreviations}

AWD: Acute watery diarrhea; DNA: Deoxyribonucleic acid; PCR: Polymerase chain reaction; PRISMAP: Preferred Reporting Items for Systematic review and Meta-Analysis Protocols; RNA: Ribonucleic acid; QPCR: Quantitative polymerase chain reaction; RT-PCR: Real-time polymerase chain reaction; RT-qPCR: Real-time reverse transcription polymerase chain reaction; MPCR: Multiplex polymerase chain reaction; CPCR: Competitive polymerase chain reaction; SmaRT-PCR: Smart real time polymerase chain reaction.

\section{Declarations}

\section{Acknowledgements}

Not applicable

\section{Authors' contributions}

Shibabaw Tadesse Gemeda (STG) deliberated of the study, and participated in its design and coordination. Involved in data extraction, critical appraisal conducting using all papers selected for inclusion in the review, drafted the manuscript and included feedback from other team members. Negasa Eshete Soboksa (NES) developed the research methods and supported in the first drafting of the manuscript. Adey Feleke Desta (AFD), and Sirak Robele Gari (SRG) conducted the review, reading and shaping of the manuscript. All authors contributed to the drafting of the protocol, read, reviewed and approved the final manuscript.

\section{Funding}

The authors have not received any funding.

\section{Availability of data and materials}

Not applicable

\section{Ethics approval and consent to participate}

Not applicable

Consent for publication

Not applicable

\section{Competing interests}

The authors declare that they have no competing interests

\section{References}


1. Potgieter N, Taonameso S, Mudau LS and, Traoré AN. Borehole water: a potential health risk to rural communities in South Africa. Water Sci Technol Water Supply |. 2018;1:1-9.

2. Haramoto E, Kitajima M, Hata A, Torrey JR, Masago Y, Sano D and, et al. A review on recent progress in the detection methods and prevalence of human enteric viruses in water. Water Res. 2018;135:168-86.

3. Titilawo Y, Obi L and, Okoh A. Occurrence of virulence gene signatures associated with diarrhoeagenic and non-diarrhoeagenic pathovars of Escherichia coli isolates from some selected rivers in South-Western Nigeria. BMC Microbiol. 2013;15:204.

4. Sadik NJ, Uprety SR, Nalweyiso A, Kiggundu N, Banadda NE, Shisler JL, et al. Quantification of multiple waterborne pathogens in drinking water, drainage channels, and surface water in Kampala, Uganda during seasonal variation. GeoHealth. 2017;:258-69. doi:10.1002/2017GH000081.

5. Heerden J van, Ehlers MM, Heim AA, Grabow WOK. Prevalence, quantification and typing of adenoviruses detected in river and treated drinking water in South Africa. J Appl Microbiol. 2005;99:234-42.

6. Tyler CR, Söffker M and, Stevens JR. Comparative Breeding and Behavioral Responses to Ethinylestradiol Exposure in Wild and Laboratory Maintained Zebrafish ( Danio rerio ) Populations. Environ Sci Technol. 2012;46:11377-83.

7. Edge TA, Khan IUH, Bouchard R, Guo J, Hill S, Locas A, et al. Occurrence of Waterborne Pathogens and Escherichia coli at Offshore Drinking Water Intakes in Lake Ontario. 2013;79:5799-813.

8. Ishii S, Kitamura G, Segawa T, Kobayashi A, Miura T, Sano D and, et al. Microfluidic Quantitative PCR for Simultaneous Quantification of Multiple Viruses in Environmental Water Samples. Am Soc Microbiol. 2014;80:7505-11.

9. Chao KK, Chao CC, Chao WL. Evaluation of Colilert-18 for Detection of Coliforms and Eschericha coli in Subtropical Freshwater. Appl Environ Microbiol. 2004;70:1242-4.

10. Schets FM, Nobel PJ, Strating S, Mooijman KA, Engels GB, Brouwer A. Comparison of methods for enumeration of total coliforms and Escherichia coli in water samples in the Netherlands. Rijksinst Voor Volksgezond En Milieu Natl Inst Public Heal Environ. 2001; January 2001:64.

11. Brenner KP, Rankin CC, Sivaganesan M, Scarping P V. Comparison of the recoveries of Escherichia coli and total coliforms from drinking water by the MI agar method and the U.S. Environmental Protection Agency-approved membrane filter method. Appl Environ Microbiol. 1996;62:203-8.

12. Harwood VJ, Pisciotta JM, Rath DF, Stanek PA, Flanery DM. Marine Bacteria Cause False-Positive Results in the Colilert-18 Rapid Identification Test for Escherichia coli in Florida Waters. 2002;68:539-44.

13. Fricker CR, Eldred BJ. Identification of coliform genera recovered from water using different technologies. Lett Appl Microbiol. 2009;49:685-8.

14. Hugenholtz P, Tyson GW. Microbiology Metagenomics. Nature. 2008;455:481-3.

15. Domingues $L$ and, Silva DM. On the track for an efficient detection of Escherichia coli in water: $A$ review on PCR-based methods. Ecotoxicol Environ Saf. 2014;113:400-11. 
16. Girones R, Ferrús MA, Alonso JL, Rodriguez-Manzano J, Calgua B, de Abreu Corrêa A, et al. Molecular detection of pathogens in water - The pros and cons of molecular techniques. Water Research. 2010;44:4325-39.

17. Denayer S, Dierick K, Botteldoorn N. Use and Validation of Real-Time PCR Methods for the Detection of Food Pathogens. Labinfo. 2010;:4-7.

18. Botes M, Kwaadsteniet M de and, Cloete TE. Application of quantitative PCR for the detection of microorganisms in water. Anal Bioanal Chem. 2012.

19. Gironesa R, Albinana-Gimeneza N, Miagostovich MP, Calguaa B, Huguet JM and, Matiac L. Analysis of adenoviruses and polyomaviruses quantified by qPCR as indicators of water quality in source and drinking-water treatment plants. Water Res. 2009;43:2011-9.

20. Utpal R, Rehan AD, Kopal J and, Sunil B. Recent developments in detection and enumeration of waterborne bacteria: a retrospective minireview. Microbiol Publ by John Wiley Sons Ltd. 2016;5:90122.

21. Szibor M and, Morawietz H. SmaRT-PCR: a novel application of competitive PCR for mRNA quantitation. Elsevier Sci Ltd. 2001;6:1366-2120.

22. Fiedoruk K, Daniluk T, Rozkiewicz D, Zaremba ML, Oldak E, Sciepuk M, et al. Conventional and molecular methods in the diagnosis of community-acquired diarrhoea in children under 5 years of age from the north-eastern region of Poland. Int J Infect Dis. 2015;37:145-51.

23. Abou Tayoun AN, Burchard PR, Malik I, Scherer A and, Tsongalis GJ. Democratizing Molecular Diagnostics for the Developing World. Am Soc Clin Pathol J Clin Pathol. 2014;141:17-24.

24. Alhamlan FS, Al-Qahtani1 AA and, Al-Ahdal MN. Recommended advanced techniques for waterborne pathogen detection in developing countries: Advanced approaches for assessing water quality. $\mathrm{J}$ Infect Dev Ctries. 2015;9:128-35.

25. Standridge J. E. coli as a public health indicator of drinking water quality. Am Water Work Assoc J. 2008;100:10,65-75.

26. Moher D, Liberati. A, Tetzlaff. J and, Altman. D. Preferred Reporting items for Systematic Reviews and Meta-Analyses: The PRISMA Statement. PLoS Med 6(6) e1000097. 2009;6:6.

27. Joshi Mohini and Deshpande J.D. Polymerase chain reaction: Methods, Priniciples and Application. Int J Biomed Res. 2011;2 (1) IJBR:81-97.

28. Moola S, Munn Z, Tufanaru C, Aromataris E, Sears K, Sfetcu R, Currie M, Qureshi R, Mattis P, Lisy K MP-F. Chapter 7: Systematic reviews of etiology and risk - Joanna Briggs Institute Reviewers' Manual. Joanna Briggs Institute Reviewer's Manual. 2017.

29. Higgins JPT, Thompson SG. Quantifying heterogeneity in a meta-analysis. Stat Med. 2002;21:153958.

30. Guyatt GH, Oxman AD, Kunz R, Falck-Ytter Y, Vist GE L, A et al. Going from evidence to recommendations. BMJ. 2008;336:1049-51. 
31. Guyatt GH, Oxman AD, Akl EA, Kunz R, Vist GE, Brozek J, et al. GRADE guidelines: 1. IntroductiondGRADE evidence profiles and summary of findings tables. J Clin Epidemiol. 2011;64:384-94.

\section{Figures}

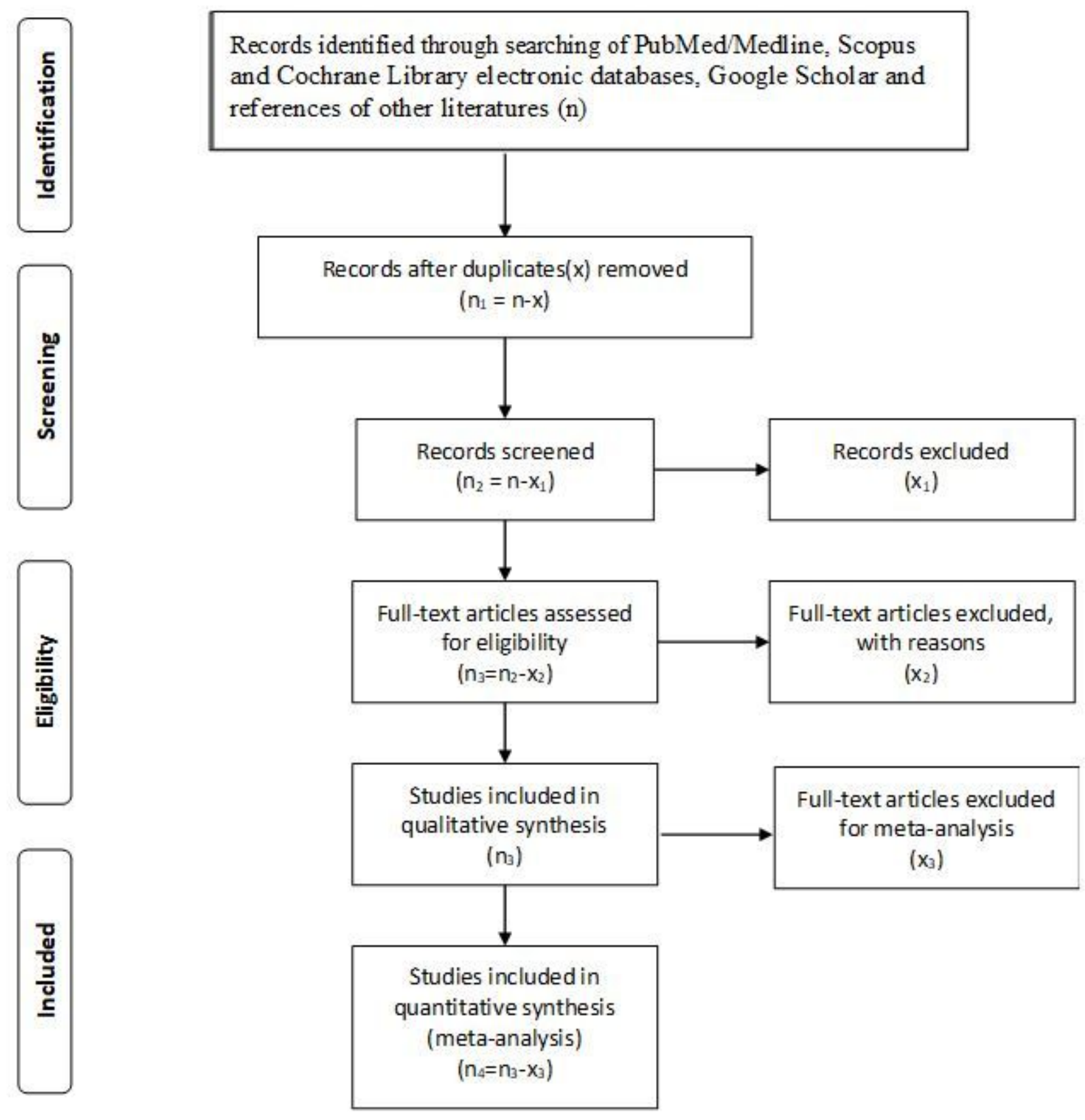

\section{Figure 1}

Study selection process of the systematic review and meta-analysis 